\title{
A Signal Method for Mobile Handheld Devices
}

\author{
Hoyoung Hwang \\ Department of Multimedia Engineering, \\ Hansung University, Korea \\ hyhwang@hansung.ac.kr
}

\begin{abstract}
A new type of digital signal method is presented for embedded systems including mobile handheld devices. A digital communication method is suggested for mobile phone and embedded devices using screen light signals that contain data such as game item, payment information, personal information, and so on. The new method provides an economic and efficient solution to mobile networking environment. We studied the solutions focusing on payment system by updating embedded software without need of additional hardware modules to mobile devices. The solutions can be used also for digital item movement between mobile phones or game devices.
\end{abstract}

Keywords: Mobile, Signal, Handheld Device, Payment

\section{Introduction}

As a result of convergence trends in technology, various functions including digital camera, game, music player which were produced and purchased as independent devices before are now integrated in a single mobile device such as smart phone or tablet. One of those functionalities is digital cash or payment system. Several mobile payment methods or systems have been developed in order to avoid the inconvenience of carrying cash or credit card. Those payment systems are mainly used for mobile phone devices and mostly for small-valued payment. However, in conventional mobile payment systems, a payment signal transmitting device and a wireless internet connection device are separately provided to the mobile phone or embedded devices. Therefore, the production cost of the mobile phone or devices increases, and the complexity and size of the mobile devices grow. In addition, there is another critical problem in that the users who want to use the mobile payment system may need to purchase a new phone or devices with additional hardware functions.

A new mobile payment method is proposed to solve the problems. The method is implemented by updating embedded software of a phone without the need of additional hardware modules to the existing mobile phone or devices. In the proposed method, a virtual mobile card containing payment-related information is loaded to the virtual machine in the mobile phone or embedded system OS. The virtual mobile card can comprise various types of payment-related information such as credit card number, membership card number, discount coupon image, online ticket, etc., which are downloaded from a control server and stored in the mobile phone or devices. The virtual mobile card information is transmitted by image by blinking the backlight of the LCD/LED panel of the mobile devices, and the transmitted information is decoded by the photo receiver which is designed for the proposed mobile payment system. The data transmission function is implemented by embedded software that controls the lightning device of a phone or handheld systems, which can be applied to the most mobile phone already in use. In addition, 
this solution can be applied to other purposes as well as payment such as digital item movement between mobile handheld devices for games or mobile commerce.

\section{Mobile Payment System}

It is believed that normally 4 or 5 credit cards are owned and carried per adult person in addition to transportation card, debit card, membership card, ID card, and etc. There is a problem in that, when the cards are lost or stolen, the owner may suffer a great deal of damages. In order to solve the inconvenience of carrying various cards, several approaches have been tried to put those card information into a phone or other embedded devices. The approaches include barcode-based solution, and IR/RF-based solution as presented in Table 1.

Some telecom companies adapted barcode type membership card into mobile phones and used it as mobile payment and membership authentication. This method is easy to implement and widely applicable to membership management applications. The weak point of this method is that the information being contained by a barcode is very limited. Moreover, if conventional laser type barcode reader devices are used, many transmission errors occur because of the reflection problem on the surface of the display device of a phone. Therefore, CCD type reader device is needed which increase the cost of system. In addition, barcode type information can be easily copied thus incurs security problem.

Table 1. Characteristics of Mobile Payment Methods

\begin{tabular}{|c|c|c|c|}
\hline & Light Signal & Barcode & IR/RF \\
\hline Additional Hardware & $\begin{array}{c}\text { No Need. } \\
\text { Use the existing } \\
\text { backlight of devices }\end{array}$ & $\begin{array}{c}\text { No Need. } \\
\text { Barcode image. }\end{array}$ & $\begin{array}{c}\text { Need. } \\
\text { RFID or smartcard chip } \\
\text { required }\end{array}$ \\
\hline Reuse existing devices & $\begin{array}{c}\text { High. } \\
\text { Adaptable to all the } \\
\text { existing phone devices }\end{array}$ & $\begin{array}{c}\text { High. } \\
\text { Adaptable to all the } \\
\text { existing phone devices }\end{array}$ & $\begin{array}{c}\text { Low. } \\
\text { Need to purchase a new } \\
\text { devices }\end{array}$ \\
\hline Recurity & $\begin{array}{c}\text { Yes. } \\
\text { security s/w module } \\
\text { needed }\end{array}$ & $\begin{array}{c}\text { No. } \\
\text { Easily copied }\end{array}$ & $\begin{array}{c}\text { High. } \\
\text { High security and large } \\
\text { volume of data }\end{array}$ \\
\hline Data Error & $\begin{array}{c}\text { Low. } \\
\text { Cheap production cost }\end{array}$ & $\begin{array}{c}\text { High. } \\
\text { Relatively expensive } \\
\text { CCD reader }\end{array}$ & $\begin{array}{c}\text { High. } \\
\text { Number of reader } \\
\text { devices increases }\end{array}$ \\
\hline Business Model \\
Scalability & $\begin{array}{c}\text { Low. } \\
\text { Allow little dust and } \\
\text { small movement }\end{array}$ & $\begin{array}{c}\text { Heflection problem } \\
\text { Reflect } \\
\text { and No movement }\end{array}$ & $\begin{array}{c}\text { Got affected by dust or } \\
\text { movement }\end{array}$ \\
\hline $\begin{array}{c}\text { Can be adapted to } \\
\text { various applications } \\
\text { based on WAP/Java }\end{array}$ & $\begin{array}{c}\text { Low. } \\
\text { Limitation of } \\
\text { applications and } \\
\text { information }\end{array}$ & $\begin{array}{c}\text { Low. } \\
\text { Additional h/w needed. } \\
\text { Good for business area } \\
\text { with high security. }\end{array}$ \\
\hline
\end{tabular}

More widely adapted mobile payment method is using IrDA or RFID technology to integrate payment related information into a phone or embedded devices. This method show high ability of processing large volume of data and multimedia applications, and provides high level of security management functions. However, the critical shortcoming of this method is that it requires additional hardware modules. Therefore, users should have specialized phones or devices with the additional hardware module instead of existing devices already in use, which makes widespread use of this method difficult.

A new mobile payment method is proposed to solve the problems of existing phone payment methods. The proposed method can be applied to the most mobile phones or devices already in use by updating embedded software without additional hardware modules. 


\section{Light Signal based Communication System}

The proposed mobile payment method transmits payment related information using the backlight of devices which is adopted in most mobile phones and embedded devices, thus eliminates the need of supplying new customized devices. The payment information using backlight signal is called virtual mobile card. The virtual mobile card based payment method has the following characteristics.

- The software application program is downloaded to the virtual machine of a phone or embedded devices.

- The optical source such as LCS backlight or keypad backlight of existing phones or devices is used for information transmission including software download and payment.

- Various types of security management solutions can be implemented by software.

- Large volume of card information data can be adopted as long as the embedded memory size allows.

- The card information can be downloaded by wireless connection immediately after the subscription.

- CRM (Customer Relationship Management) and other applications can be easily applicable by coordination with phone number information.

- The cost of optical reader device is relatively cheap compared with CCD type readers or IR/RF type readers.

The mobile communication system using a light signal consists of three parts: a mobile phone for outputting a predetermined light signal; a photo receiver for converting the received light signal to an electrical signal, encrypting the electrical signal, and outputting the encrypted electrical signal as an output signal; and the control server for authenticating payment by using the output signal of the photo receiver.

Figure 1 is a block diagram showing a mobile phone payment system. The system comprises a mobile phone, a photo receiver, and a control server.

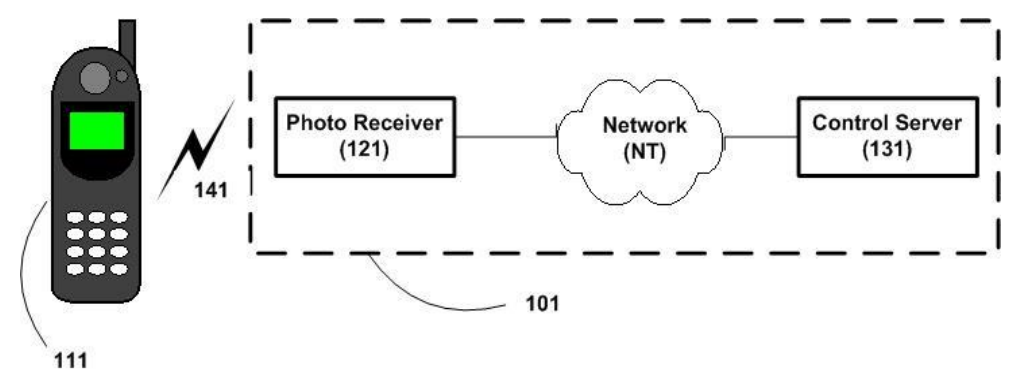

Figure 1. Block Diagram Showing a Mobile Signaling System

The photo receiver comprises a photo sensor and a data error determination unit. The photo sensor of the photo receiver receives the light signal and converts the light signal into an electrical signal. The received light signal includes the payment related information. The data error determination unit determines whether the light signal is transmitted without error from the mobile phone to the photo receiver. If the light signal is determined to be transmitted without error form the mobile phone to the photo receiver, the photo receiver transmits the electrical signal from the photo sensor through a network NT to the control server. If not, the photo receiver displays a message indicating that there is an error in the transmitted payment-related information, so that the user may transmit the light signal corresponding the payment-related information again.

The control server compares the transmitted payment-related information with the associated information stored in the control server. If the transmitted payment-related 
information is identical to the associated information stored in the control server, the control server authenticates the user's payment by using the payment-related information, which includes credit card information or E-money information of the user, as described above. The control server sends a short text message indicating the result of payment to the user's mobile phone through Short Message Service server. If the transmitted payment-related information is not identical to the associated information stored in the control server, the control server sends to the user's mobile phone through Short Message Service server a short text message indicating that the transmitted payment-related information is not correct. In addition, the control sever transmits the result of the payment to the photo receiver in order to inform a manager of the photo receiver of the result of the payment. If the payment is not authenticated, the user may transmit the payment-related information again to perform the aforementioned payment process.

\subsection{Mobile Phone Signal}

Figure 2 is a waveform diagram showing pulses of a light signal generated by a backlight of a mobile phone. The light signal is partitioned into a starting frame $t 1$, an information frame $\mathrm{t} 2$, and an ending frame $\mathrm{t} 3$. The starting frame $\mathrm{t} 1$ comprises a plurality of short pulses to indicate the starting point of the light signal. The information frame $\mathrm{t} 2$ comprises a plurality of pulses generated based on the payment-related information. The ending frame $\mathrm{t} 3$ comprises a plurality of pulses to indicate the ending point of the light signal. The information frame $\mathrm{t} 2$ is constructed with a plurality of information bits t 11 and t12. The information frame $\mathrm{t} 2$ represents a piece of information with the plurality of information bits $\mathrm{t} 11$ and $\mathrm{t} 12$. For example, when the information frame $\mathrm{t} 2 \mathrm{is}$ " 1010 ", the information frame $\mathrm{t} 2$ is constructed with two " 1 " bits and two " 0 " bits. In addition, the information bit $\mathrm{t} 11$ is partitioned into a starting bit $\mathrm{t} 21$, a main bit $\mathrm{t} 22$, a parity bit $\mathrm{t} 23$, and a stop bit $\mathrm{t} 24$. The starting bit $\mathrm{t} 21$ indicates the starting point of the information bit $\mathrm{t} 11$. The main bit $\mathrm{t} 22$ represents real information with " 1 " or " 0 " bit. The parity bit $\mathrm{t} 23$ is used to determine whether or not the light signal is transmitted without error. The stop bit t24 indicates the ending point of the information bit t11.

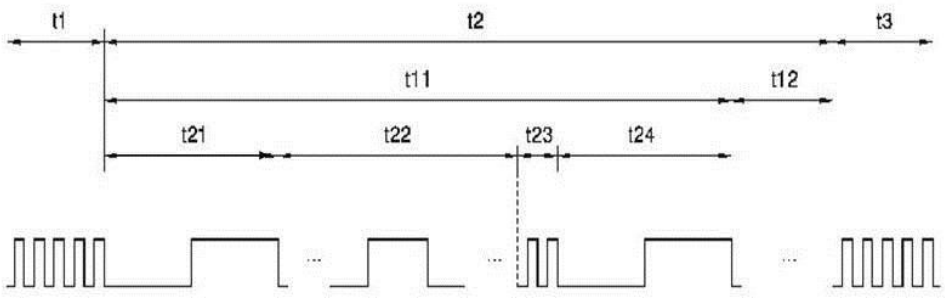

\section{Figure 2. Waveform Diagram Showing Pulses of a Signal Generated by a Mobile Phone}

\subsection{Photo Receiver}

A photo receiver decodes a light signal from a mobile phone, converting the light signal to an electrical signal, and transmitting the electrical signal to a control server. The photo receiver comprising: a photo sensor for receiving a light signal from a mobile phone, and converting the light signal into an electrical signal; a data error determination unit for receiving the electrical signal of the photo sensor and determining an error of the electrical signal; a control unit for transmitting the electrical signal of the photo sensor to the data error determination unit, outputting a control signal based on the result of the error determination by data error determination unit, and outputting the electrical signal if the electrical signal has no error; a display unit for displaying presence or absence of the error of the light signal in response to the control signal; and a communication unit for transmitting the electrical signal from the control unit to the control server. 
Figure 3 is a block diagram showing the photo receiver. The photo receiver may further comprise an encryption unit for encrypting the electrical signal transmitted from the control unit, and a communication unit for transmitting the encrypted signal by the encryption unit to the control server. The display unit may comprise at least one of a third lighting device which is turned on, if a signal indicating that the payment-related information transmitted from the mobile phone is correct is transmitted from the control server, and a forth lighting device is turned on, if not, and the control unit may receive a predetermined signal from the control server via the communication unit and control the operation of the display unit in accordance with the received signal.

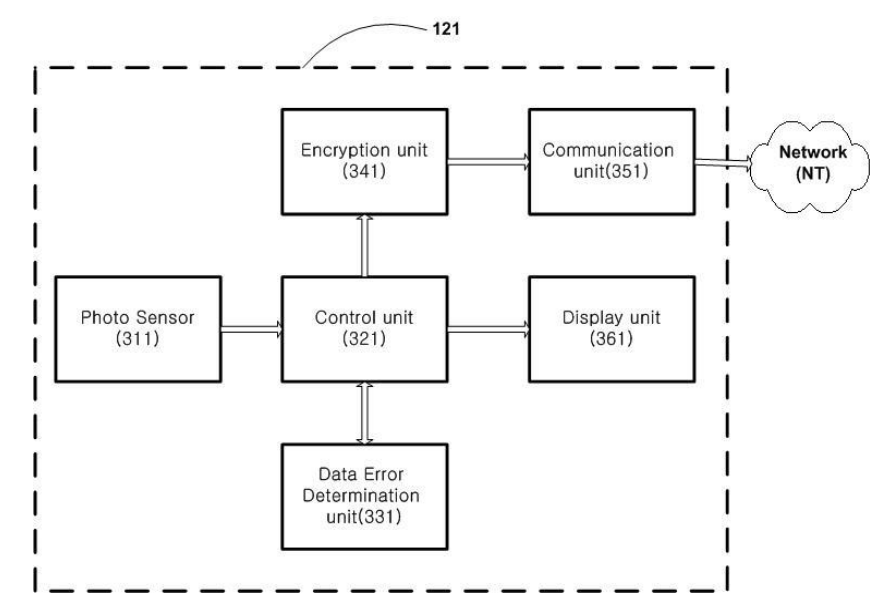

Figure 3. Block Diagram Showing a Photo Receiver

\subsection{Control Server}

Figure 4 is a block diagram showing the control server in detail. The control server comprises a data storage unit, a decoder, a data comparator, a payment authentication unit, and a communication unit. The data storage unit stores personal information of a subscribed user. The communication unit transmits a result of the payment authentication to the mobile phone.

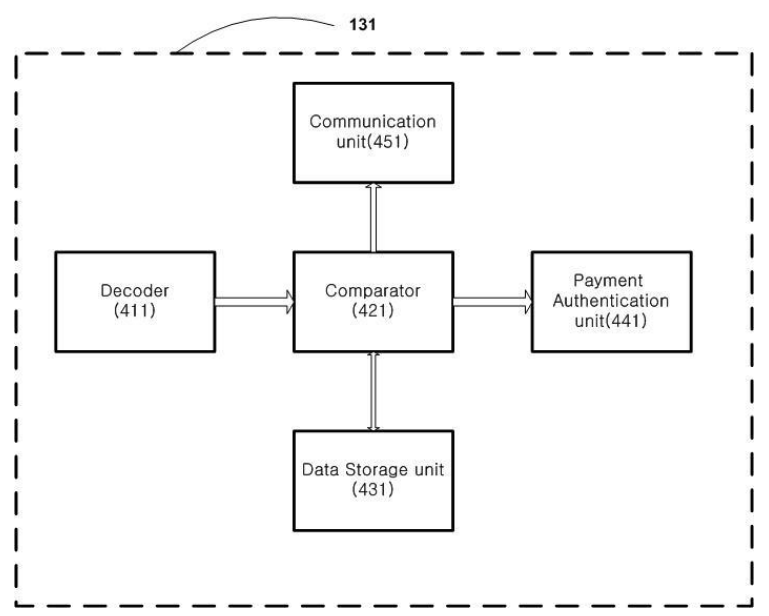

Figure 4. Block Diagram Showing a Control Server

The mobile payment method is comprised of the following steps: (a) downloading the payment-related information of the user from the control server to the mobile phone and storing the payment-related information in the mobile phone; (b) converting the paymentrelated information into a binary code data in response to the user's request; (c) generating 
a light signal having a series of pulses by turning on and off a backlight of the mobile phone based on the binary code data and transmitting the light signal to the photo receiver; (d) receiving the light signal in the photo receiver, converting the received light signal into an electrical signal and transmitting the electrical signal from the photo receiver to the control server; (e) receiving the electrical signal, comparing paymentrelated information contained in the electrical signal with the payment-related information of the user stored in the control server and determining whether or not the transmitted payment-related information is correct; ( $f$ ) if the transmitted payment-related information is correct, authenticating the user's payment; and $(\mathrm{g})$ transmitting the result of the determination of the step (e) from the control server to at least one of the mobile phone and the photo receiver. The step (b) may further comprise a step of encrypting the binary code data, and the step (e) may further comprise a step of decoding the received electrical signal.

Figure 5 is a flowchart showing a mobile signaling system for payment applications.

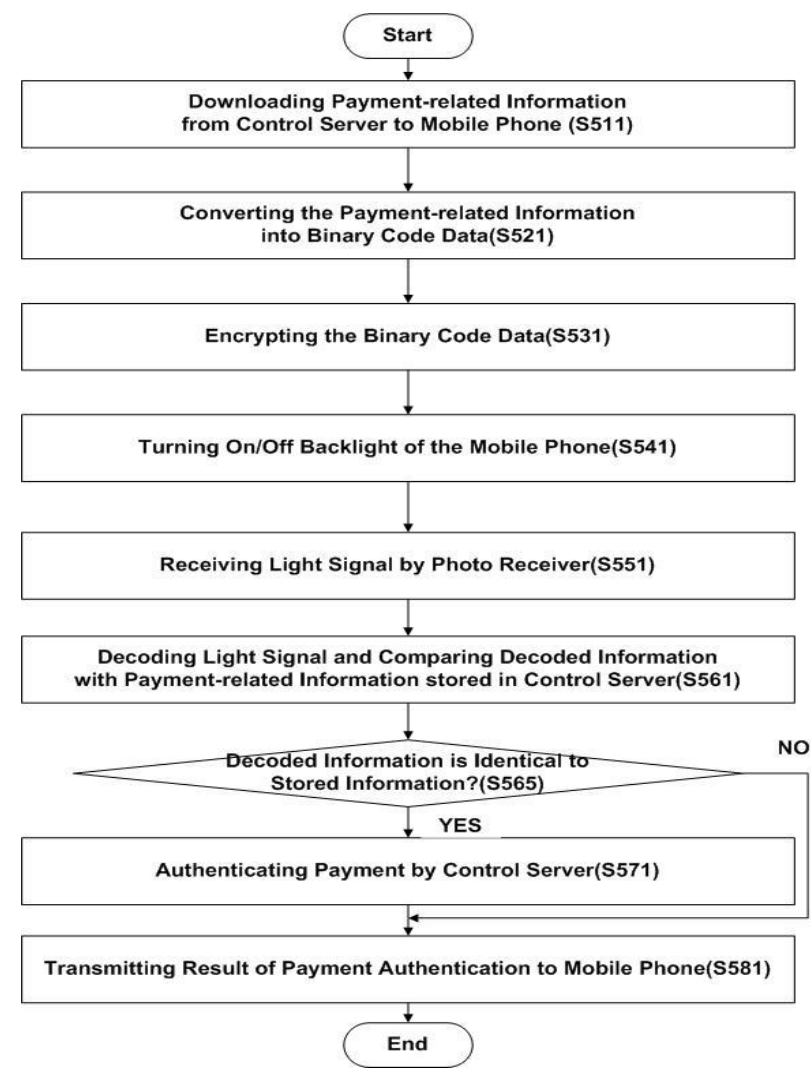

Figure 5. Flowchart Showing a Mobile Payment Procedure

\section{Conclusion}

A mobile payment system was proposed for mobile phone or embedded devices. The system consists of mobile device, photo receiver, and control server. The payment related information is downloaded from the control server to the user device. The new method provides an economic and efficient solution to mobile payment system using light signal. It is possible for a user of a mobile phone or handheld device to make payments by using his or her device without carrying paper money or a credit card. In addition, it is possible to make payments by using a mobile payment system being implemented by updating embedded software of the mobile phone or devices without additional hardware modules. Therefore, it is possible to reduce production costs of mobile phones and mobile payment systems. 
The proposed method can be applied for digital item movement between mobile phones or game devices, and also for authentication in SNS based games or LBS services.

\section{Acknowledgement}

This work was financially supported by Hansung University

\section{References}

[1] L. Antovski, M. Gusev, "M-payments", Proceedings of International Conference on Information Technology Interfaces, (2003).

[2] N. Delic, A. Vukasinovic, "Mobile Payment Solution - Symbiosis between Banks, Application Service Providers and Mobile Network Operators", Proceedings of International conference on Information Technology: New Generations, (2006), pp. 346-350.

[3] J. Gao, K. Edunuru, J. Cai, S. Shim, "A Peer-to-Peer Wireless Payment System", Proceedings of International Conference on Mobile Commerce and Services, (2005).

[4] O. Lee, "Sound-based Mobile Payment System", Proceedings of International Conference on Web Services, (2004).

[5] N. Mallat, V. K. Tuunainen, "Merchant Adoption of Mobile Payment Systems", Proceedings of International Conference on Mobile Business, (2005).

[6] K. Pousttchi, M. Zenker, "Current Mobile Payment Procedures on the German Market from the View of Customer Requirements", Proceedings of Workshop on Database and Expert Systems and Applications, (2003).

[7] X. Wu, O. Dandash and P. D. Le, "The Design and Implementation of a Smartphone Payment System based on Limited-used Key Generation Scheme", Proceedings of International Conference on Information Technology: New Generation, (2006).

[8] X. Zheng, D. Chen, "Study of Mobile Payments System", Proceedings of International Conference on E-Commerce, (2003).

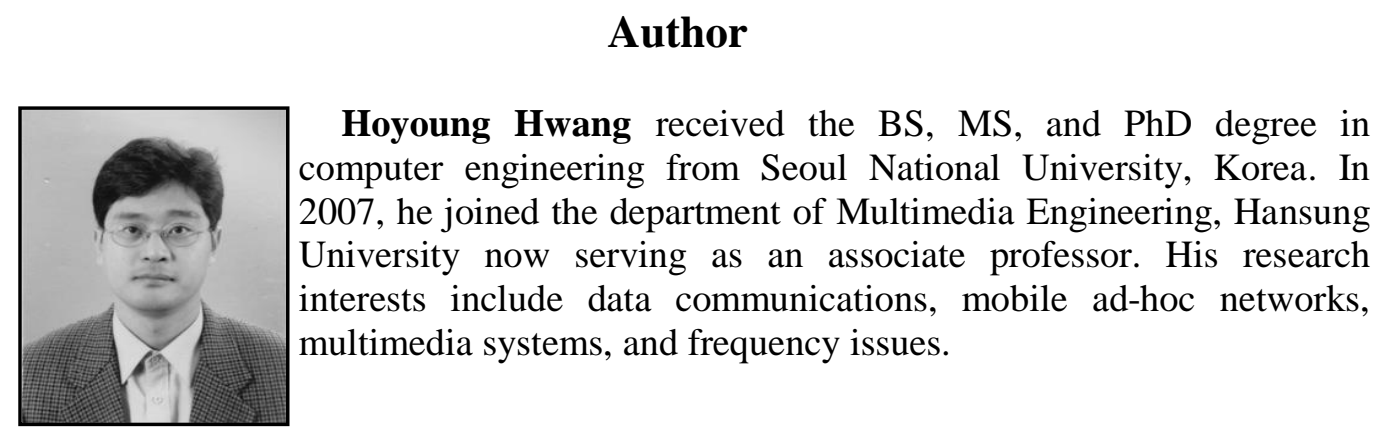


International Journal of Multimedia and Ubiquitous Engineering

Vol. 10, No. 3 (2015) 